\title{
Signatures of quantum fluctuations in the Dicke model by means of Rényi uncertainty
}

\author{
M. Calixto \\ Departamento de Matemática Aplicada, Universidad de Granada, Fuentenueva s/n, 18071 Granada, Spain \\ Á. Nagy \\ Department of Theoretical Physics, University of Debrecen, H-4010 Debrecen, Hungary \\ I. Paradela and E. Romera \\ Departamento de Física Atómica, Molecular y Nuclear and Instituto Carlos I de Física Teórica y Computacional, \\ Universidad de Granada, Fuentenueva s/n, 18071 Granada, Spain
}

(Dated: February 2, 2012)

\begin{abstract}
Rényi uncertainty relations are shown to have significant importance in quantum phase transitions. They detect the quantum phase transition in the Dicke model. The Rényi entropy sum is more adequate in describing quantum fluctuations than the standard variance products.
\end{abstract}

\section{INTRODUCTION}

Uncertainty relations play a central role in quantum physics. The Heisenberg uncertainty principle is generally expressed in terms of the familiar variance-based uncertainty relation. Uncertainty principle can alternatively be formalized via entropic uncertainty relation [1$3]$ that proved to be very useful in several situations [111]. Quantum phase transitions (QPT) result from the variation of quantum fluctuations [12]. Therefore uncertainty relations are essential in studying QPT. Variancebased uncertainty relations have been often applied. In this paper we argue that Rényi uncertainty relations have significant importance in quantum phase transitions. As an illustration the Dicke model is presented. The Rényi entropy sum has an abrupt change at the transition point, though it remains constant in both phases. Whereas, the variance products are divergent in the superradiant phase.

Rényi entropy [13] of order $\mu$ for a $D$ dimensional probability density function $f\left(r_{1}, \ldots, r_{D}\right)$ normalized to one is defined by

$$
R_{f}^{\mu} \equiv \frac{1}{1-\mu} \ln \int f^{\mu}(\mathbf{r}) d \mathbf{r}, \quad \text { for } \quad 0<\mu<\infty, \quad \mu \neq 1,
$$

where $\mathbf{r}$ stands for $\left(r_{1}, \ldots, r_{D}\right)$. Rényi entropy can be considered a one-parameter extension of Shannon entropy [14] as the Rényi entropy tends to the Shannon entropy

$$
S_{f}=-\int f(\mathbf{r}) \ln f(\mathbf{r}) d \mathbf{r}
$$

when $\mu \rightarrow 1$. Rényi entropy has been applied in several fields of quantum physics, such as quantum entanglement [15], quantum communication protocols [16], quantum correlations [17], localization properties [18], quantum revivals [19] and atomic physics [20-23].

Suppose that the probability distribution $\rho(\mathbf{r})$ can be associated with a wave function $\psi(\mathbf{r})$ as $\rho(\mathbf{r})=|\psi(\mathbf{r})|^{2}$. The probability distribution $\gamma(\mathbf{p})$, on the other hand, is given by the momentum space wave function $\phi(\mathbf{p})$, the
Fourier transform of the wave function $\psi(\mathbf{r})$, as $\gamma(\mathbf{p})=$ $|\phi(\mathbf{p})|^{2}$. An uncertainty relation for the Rényi entropy sum can be found in the literature directly based on the Hausdorff-Young inequality [24-26]:

$$
\begin{gathered}
R_{\rho}^{\mu}+R_{\gamma}^{\nu} \geq g(\mu, \nu), \quad \frac{1}{\mu}+\frac{1}{\nu}=2 \\
g(\mu, \nu)=\frac{D}{2}\left[\frac{1}{\mu-1} \ln \left(\frac{\mu}{\pi}\right)+\frac{1}{\nu-1} \ln \left(\frac{\nu}{\pi}\right)\right],
\end{gathered}
$$

where $D$ is the dimension. This uncertainty relation reaches the Shannon entropic uncertainty relation [2729]

$$
S_{\rho}+S_{\gamma} \geq D \ln (e \pi)
$$

in the limit $\mu \rightarrow 1$. Eq. (3) is saturated by Gaussian distribution functions, that is, the Rényi uncertainty relation is sharp.

We mention in passing that the Rényi entropy sum is the sum obtained from the phase space marginal distributions. Further interesting inequalities can be found in [30].

In classical phase transitions there is an abrupt change in the physical properties of a system as a parameter (normally the temperature), being responsible for the transition, changes. The phenomena are due to classical fluctuations (thermal fluctuations in case the temperature was the parameter). Quantum phase transitions, on the other hand, occur at zero temperature. The quantum systems are at their ground states and the abrupt change in the physical properties are induced by a parameter of the system. The Hamiltonian can be written as $H=H(\lambda)=H_{0}+\lambda V$ with $H_{0}$ integrable. At the critical point $\lambda=\lambda_{c}$, there is an abrupt change in the symmetry of the ground-state wavefunction [12].

The Rényi entropy is a functional of the probability density. As the symmetry of the ground-state wavefunction changes, both the position and the momentum space probability densities change. Therefore there should be 
an abrupt change in the Rényi entropy sum, too. Entropic uncertainty relation provides (see [31] and references therein) a refined version of the Heisenberg uncertainty relation:

$$
\Delta x \Delta p_{x} \geq \frac{1}{2} \exp \left[S_{\rho}+S_{\gamma}-1-\ln \pi\right] \geq \frac{1}{2} .
$$

It gives a stronger bound for the variance product than the standard $\frac{1}{2}$. That is, the relation with the Shannon entropy sum provides a more useful form of the uncertainty relation than the one containing the variance product. We have shown that the description of the quantum phase transition in terms of the entropic uncertainty relation turns out to be more suitable than in terms of the standard variance-based uncertainty relation [32]. The importance of entropic uncertainty measure for fluctuations has recently been emphasized [33].

In this work we will show that the uncertainty relation for the Rényi entropy sum gives a fresh insight into quantum fluctuations. To illustrate it we selected the Dicke model that proved to be very useful in studying quantum optical [34-38], chaotic [38, 39] or entanglement [40] properties. It has been realized with a superfluid gas in an optical cavity [41] and the spontaneous symmetry breaking has been observed recently [42]. There is a QPT in the $N \rightarrow \infty$ limit. It has recently been shown that there is an abrupt change in the Rényi entropy at the transition point [43] and that the transition is marked by the relative complexity measure [44].

\section{RÉNYI UNCERTAINTY AND QPT IN THE DICKE MODEL}

Consider an ensemble of $N$ two-level atoms with levelsplitting $\omega_{0}$. The single-mode Dicke Hamiltonian has the form

$$
H=\omega_{0} J_{z}+\omega a^{\dagger} a+\frac{\lambda}{\sqrt{2 j}}\left(a^{\dagger}+a\right)\left(J_{+}+J_{-}\right),
$$

where $J_{z}, J_{ \pm}$are the angular momentum operators for a pseudospin of length $j=N / 2$. $a$ and $a^{\dagger}$ are the bosonic operators of the field (the bosonic mode has a frequency $\omega)$. In the thermodynamic limit, where the number of atoms becomes infinite $(N, j \rightarrow \infty)$, there is a QPT at a critical value of the atom-field coupling strength $\lambda_{c}=$ $\frac{1}{2} \sqrt{\omega \omega_{0}}$. There are two phases: normal phase $\left(\lambda<\lambda_{c}\right)$ and superradiant phase $\left(\lambda>\lambda_{c}\right)$.

Let us consider a basis set $\{|n\rangle \otimes|j, m\rangle\}$ of the Hilbert space, with $\{|n\rangle\}_{n=0}^{\infty}$ the number states of the field and $\{|j, m\rangle\}_{m=-j}^{j}$ the so called Dicke states of the atomic sector. To solve numerically the eigenproblem we have to diagonalize the matrix

$$
\left\langle n^{\prime}, j^{\prime}, m^{\prime}|H| n, j, m\right\rangle=\left(n \omega+m \omega_{0}\right) \delta_{n^{\prime}, n} \delta_{m^{\prime}, m}+
$$

$\frac{\lambda}{\sqrt{2 j}}\left(\sqrt{n+1} \delta_{n^{\prime}, n+1}+\sqrt{n} \delta_{n^{\prime}, n-1}\right)(\sqrt{j(j+1)-m(m+1)}$

$$
\left.\times \delta_{m^{\prime}, m+1}+\sqrt{j(j+1)-m(m-1)} \delta_{m^{\prime}, m-1}\right)
$$

and for this purpose we truncate the bosonic Hilbert space to a finite dimension $n_{c}$ large enough to have convergence in the solution (see [45] for a detailed study of the numerical problem).

We shall make use of the Holstein-Primakoff representation [46] of the angular momentum operators $J_{ \pm}, J_{z}$ in terms of the bosonic operators, $\left[b, b^{\dagger}\right]=1$, given by:

$$
\begin{gathered}
J_{+}=b^{\dagger} \sqrt{2 j-b^{\dagger} b}, \quad J_{-}=\sqrt{2 j-b^{\dagger} b} b \\
J_{z}=\left(b^{\dagger} b-j\right) .
\end{gathered}
$$

For high values of $j$ (and fixed $b^{\dagger} b$ ), we can approximate $J_{+} \simeq \sqrt{2 j} b^{\dagger}$ and $J_{-} \simeq \sqrt{2 j} b$, so that the atomic sector can be practically described by an harmonic oscillator, just like the field sector. Introducing then position and momentum operators for the two bosonic modes as usual:

$$
\begin{aligned}
& X=\frac{1}{\sqrt{2 \omega}}\left(a^{\dagger}+a\right), \quad P_{X}=i \sqrt{\frac{\omega}{2}}\left(a^{\dagger}-a\right), \\
& Y=\frac{1}{\sqrt{2 \omega_{0}}}\left(b^{\dagger}+b\right), \quad P_{Y}=i \sqrt{\frac{\omega_{0}}{2}}\left(b^{\dagger}+b\right),
\end{aligned}
$$

the wave function in position representation can be represented by

$$
\begin{aligned}
\psi(x, y)= & \frac{\sqrt{\omega \omega_{0}}}{\sqrt{\pi}} e^{-\frac{1}{2}\left(\omega x^{2}+\omega_{0} y^{2}\right)} \sum_{n=0}^{n_{c}} \sum_{m=-j}^{j} c_{n m}^{(j)} \\
& \times \frac{H_{n}(\sqrt{\omega} x) H_{j+m}\left(\sqrt{\omega_{0}} y\right)}{2^{(n+m+j) / 2} \sqrt{n !(j+m) !}}
\end{aligned}
$$

where we have made use of the definition of

$$
\begin{aligned}
\langle x \mid n\rangle & =\sqrt{\omega} e^{-\frac{1}{2} \omega x^{2}} \frac{H_{n}(\sqrt{\omega} x)}{\sqrt{2^{n} n ! \sqrt{\pi}}} \\
\langle y \mid j, m\rangle & =\sqrt{\omega_{0}} e^{-\frac{1}{2} \omega_{0} y^{2}} \frac{H_{j+m}\left(\sqrt{\omega_{0}} y\right)}{\sqrt{2^{(j+m)}(j+m) ! \sqrt{\pi}}},
\end{aligned}
$$

in terms of Hermite polynomials of degree $n$ and $j+$ $m$, respectively. This is a very convenient representation that has already been used in Ref. [38]. In the same way, the wave function in momentum representation can be written as:

$$
\begin{aligned}
\phi\left(p_{x}, p_{y}\right)= & \frac{1}{\sqrt{\pi}} e^{-\frac{1}{2}\left(\frac{p_{x}^{2}}{\omega}+\frac{p_{y}^{2}}{\omega_{0}}\right)} \sum_{n=0}^{n_{c}} \sum_{m=-j}^{j}(-i)^{n+m+j} c_{n m}^{(j)} \\
& \times \frac{H_{n}\left(p_{x} / \sqrt{\omega}\right) H_{j+m}\left(p_{y} / \sqrt{\omega_{0}}\right)}{2^{(n+m+j) / 2} \sqrt{n !(j+m) !}}
\end{aligned}
$$

where we have taken into account that the Fourier transform of $e^{-a^{2} x^{2} / 2} H_{n}(a x)$ is given by $(-i)^{n} e^{-p^{2} /\left(2 a^{2}\right)} H_{n}(p / a)$.

Figs. 1(a) and (b) present the Rényi uncertainty sum $R_{\rho}^{\mu}+R_{\gamma}^{\nu}$ for values of the parameter $\lambda \in[0,1]$ and for $(\mu, \nu)$ equal to $(2,2 / 3)$ and $(2 / 3,2)$, respectively. In 

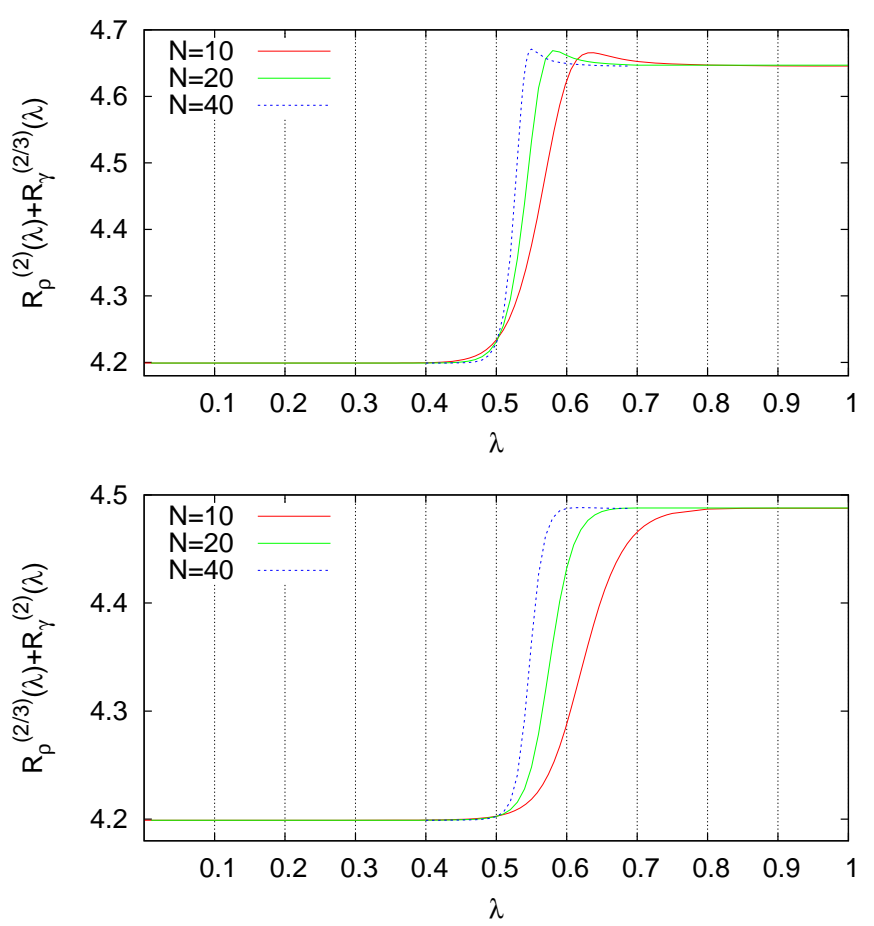

FIG. 1. Numerical results for Rényi uncertainty entropy sum $R_{\rho}^{\mu}+R_{\gamma}^{\nu}$ for the ground-state for $N=10,20,40$ and $\omega_{0}=$ $\omega=1\left(\lambda_{c}=0.5\right)$ and for $(\mu, \nu)$ equal to $(2,2 / 3)$ (top) and $(2 / 3,2)$ (bottom).
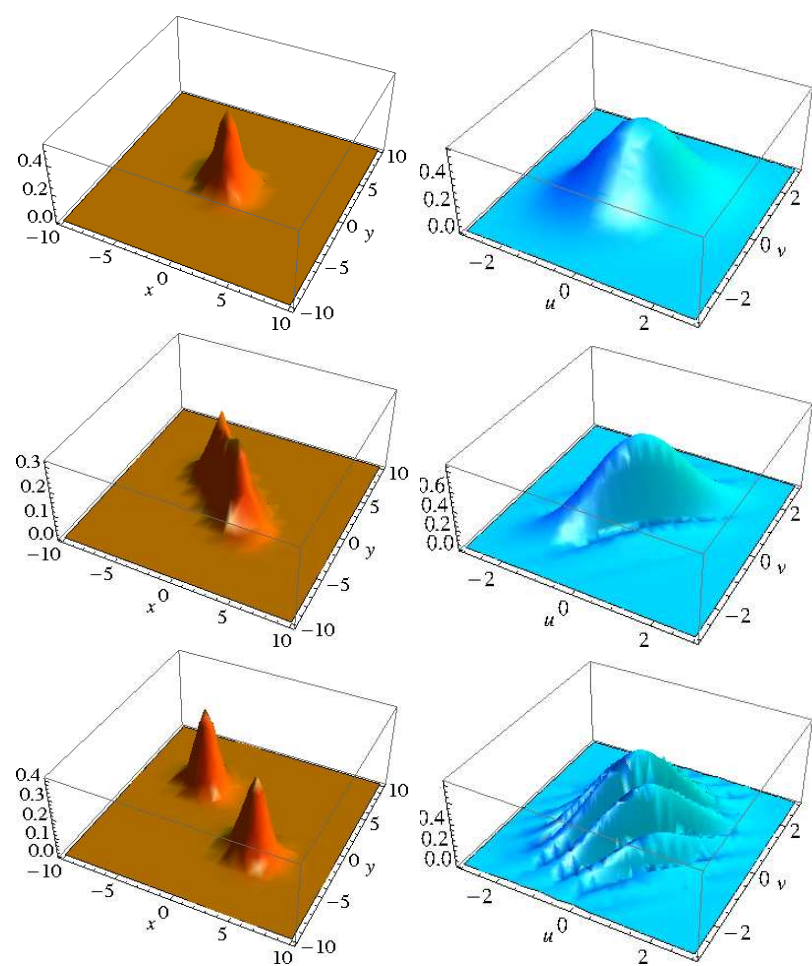

FIG. 2. Ground state density function in position space (left) and momentum space (right) for different values of $\lambda$ (from top to bottom $\lambda=0.3, \lambda=0.55$ and $\lambda=0.7$ ) for $\omega_{0}=\omega=1$ and $N=20$.
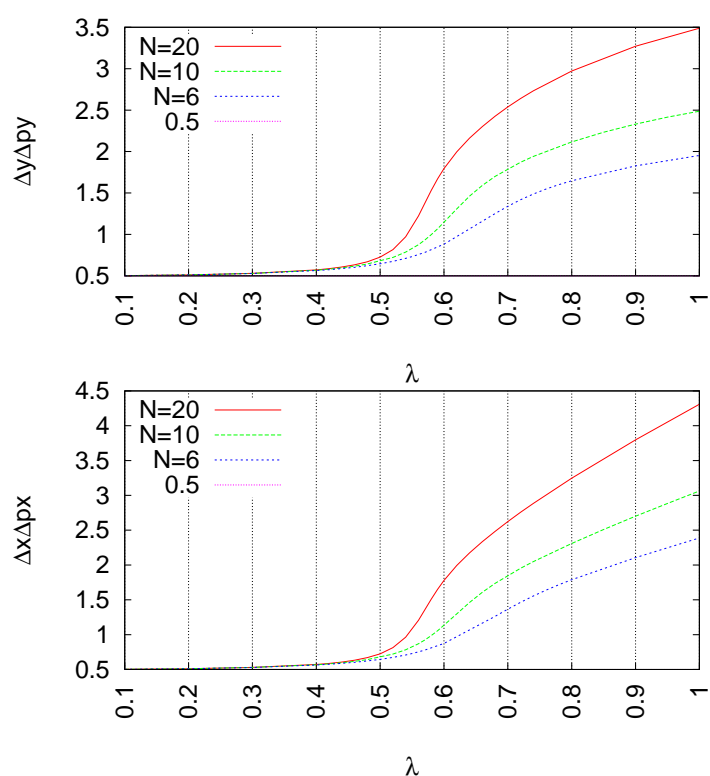

FIG. 3. Variance products $\Delta x \Delta p_{x}$ and $\Delta y \Delta p_{y}$ for the ground state for $N=6,10$ and 20 and $\omega_{0}=\omega=1$.

this case the uncertainty relation (3) is verified with $g(\mu, \nu)=\ln \left(3^{3} \pi^{2} / 2^{2}\right) \approx 4.199$. We can see that the uncertainty relation reaches about the minimum value for $\lambda<\lambda_{c}$ (normal phase). In the normal phase the position (momentum) space density function is a Gaussianlike one centered at the origin, as we can see in figure 2 left (right) top panel. The uncertainty relation reaches a constant value $\ln \left(54 \sqrt{\pi} \Gamma(7 / 6)^{3} / \Gamma(5 / 3)^{3}\right) \approx 4.64$ for values of $\lambda>\lambda_{N} \geq \lambda_{c}$, where $\lambda_{N} \rightarrow \lambda_{c}$ as $N \rightarrow \infty$ (see later on equations (24) and (25) for general $\mu, \nu)$. This situation corresponds to a position (momentum) density function composed by two subpackets moving away from each other into different quadrants in the plane as we can see in the left bottom panel of figure 2 (by a modulated Gaussian-like packet seen in the right bottom panel of figure 2). The transition from the first situation to the second one is around $\lambda=\lambda_{c}$ and it is the more sudden the greater the number of particles are (see Figure 1).

The variance products $\Delta x \Delta p_{x}$ and $\Delta y \Delta p_{y}$ are also shown (Figure 3). Calculations were performed with $\omega_{0}=\omega=1$, that is, $\lambda_{c}=0.5$. We can see that for values $\lambda<\lambda_{c}$ the uncertainty relations reach the value $1 / 2$ (that is, saturate the uncertainty relation (6)) growing as $\lambda$ increases from $\lambda_{c}$ and as $N$ increases, such that uncertainty relations diverge for $\lambda \geq \lambda_{c}$ as $N$ diverges.

The most important conclusion for this analysis is that the Rényi uncertainty relations take into account the quantum fluctuations or wave-packet uncertainty (as the entropic uncertainty does [32]) whereas that the variance uncertainty take into account not only the quantum fluctuations but the relative position of the wave-packets. 


\section{VARIATIONAL SCHRÖDINGER'S CAT-STATES AND THE THERMODYNAMIC LIMIT}

Now we present analytical expressions for uncertainty relations using trial states expressed in terms of "symmetry-adapted coherent states" introduced by Castaños et al. [47], which turn out to be an excellent approximation to the exact quantum solution of the ground $(+)$ and first excited $(-)$ states of the Dicke model.

Let us denote by

$$
\begin{aligned}
& |\alpha\rangle=e^{-|\alpha|^{2} / 2} e^{\alpha a^{\dagger}}|0\rangle=e^{-|\alpha|^{2} / 2} \sum_{n=0}^{\infty} \frac{\alpha^{n}}{\sqrt{n !}}|n\rangle, \\
& |z\rangle=\left(1+|z|^{2}\right)^{-j} e^{z J_{+}}|j,-j\rangle= \\
& \left(1+|z|^{2}\right)^{-j} \sum_{m=-j}^{j}\left(\begin{array}{c}
2 j \\
j+m
\end{array}\right)^{1 / 2} z^{j+m}|j, m\rangle
\end{aligned}
$$

the standard (canonical) and spin- $j$ coherent states for the photon and the particle sectors, respectively. Using the direct product $|\alpha, z\rangle \equiv|\alpha\rangle \otimes|z\rangle$ as a ground-state ansatz, one can easily compute the mean energy

$$
\begin{aligned}
& \mathcal{H}(\alpha, z)=\langle\alpha, z|H| \alpha, z\rangle \\
& =\omega|\alpha|^{2}+j \omega_{0} \frac{|z|^{2}-1}{|z|^{2}+1}+\lambda \sqrt{2 j}(\alpha+\bar{\alpha}) \frac{\bar{z}+z}{|z|^{2}+1} \\
& =\frac{\omega}{2}\left(q^{2}+p^{2}\right)-\omega_{0} j \cos \theta+\sqrt{4 j} \lambda q \sin \theta \sin \phi,
\end{aligned}
$$

which defines a four-dimensional 'energy surface'; we have used quadratures $\alpha=\frac{1}{\sqrt{2}}(q+i p)$ and stereographic projection $z=\tan \left(\frac{\theta}{2}\right) e^{i \phi}$ coordinates in the last equality for later convenience. Minimizing with respect to these four coordinates gives the critical points:

$$
\begin{aligned}
& \alpha=\alpha_{0}= \begin{cases}0, & \text { if } \lambda<\lambda_{c} \\
-\sqrt{2 j} \sqrt{\frac{\omega_{0}}{\omega}} \frac{\lambda}{\lambda_{c}} \sqrt{1-\left(\frac{\lambda}{\lambda_{c}}\right)^{-4}}, & \text { if } \lambda \geq \lambda_{c}\end{cases} \\
& z=z_{0}= \begin{cases}0, & \text { if } \lambda<\lambda_{c} \\
\sqrt{\frac{\frac{\lambda}{\lambda_{c}}-\left(\frac{\lambda}{\lambda_{c}}\right)^{-1}}{\lambda_{c}}+\left(\frac{\lambda}{\lambda_{c}}\right)^{-1}} & \text { if } \lambda \geq \lambda_{c}\end{cases}
\end{aligned}
$$

Note that $\alpha_{0}$ and $\beta_{0}$ are real, so that $p_{0}=0=\phi_{0}$.

Although the direct product $|\alpha, z\rangle$ gives a good variational approximation to the ground state mean energy in the thermodynamic limit $j \rightarrow \infty$, it does not capture the correct behavior for other ground state properties sensitive to the parity symmetry $\hat{\Pi}$ of the Hamiltonian (7) like, for instance, uncertainty measures. This is why parity-symmetry adapted coherent states (which turn out to be a special kind of 'Schrödinger's cat-states') are introduced. Indeed, a far better variational description of the ground (resp. first-excited) state is given in terms of the even-(resp. odd)-parity CSs

$$
|\alpha, z, \pm\rangle=\frac{|\alpha\rangle \otimes|z\rangle \pm|-\alpha\rangle \otimes|-z\rangle}{\mathcal{N}_{ \pm}(\alpha, z)},
$$

obtained by applying projectors of even and odd parity to the direct product $|\alpha\rangle \otimes|z\rangle$. Here

$$
\begin{aligned}
\mathcal{N}_{ \pm}(\alpha, z) & =\sqrt{2}\left(1 \pm e^{-2|\alpha|^{2}}\left(\frac{1-|z|^{2}}{1+|z|^{2}}\right)^{2 j}\right)^{1 / 2} \\
& =\sqrt{2}\left(1 \pm e^{-\left(p^{2}+q^{2}\right)}(\cos \theta)^{2 j}\right)^{1 / 2}
\end{aligned}
$$

is a normalization factor. The new energy surface is now:

$$
\begin{aligned}
\mathcal{H}_{ \pm}(\alpha, z) & =\langle\alpha, z, \pm|H| \alpha, z, \pm\rangle \\
& =\frac{\mathcal{H}(\alpha, z) \pm\langle\alpha, z|H|-\alpha,-z\rangle}{\mathcal{N}_{ \pm}^{2} / 2},
\end{aligned}
$$

with non-diagonal elements

$$
\begin{aligned}
& \langle\alpha, z|H|-\alpha,-z\rangle=e^{-2|\alpha|^{2}}\left(\frac{1-|z|^{2}}{1+|z|^{2}}\right)^{2 j} \times \\
& \left(\omega|\alpha|^{2}-j \omega_{0} \frac{1+|z|^{2}}{1-|z|^{2}}+\lambda \sqrt{2 j}(\alpha-\bar{\alpha}) \frac{z-\bar{z}}{1-|z|^{2}}\right) \\
& =e^{-\left(p^{2}+q^{2}\right)}(\cos \theta)^{2 j} \times \\
& \left(\frac{\omega}{2}\left(q^{2}+p^{2}\right)-\omega j(\cos \theta)^{2 j-1}-\sqrt{4 j} \lambda p \sin \theta \cos \phi\right) .
\end{aligned}
$$

The more involved structure of $\mathcal{H}_{ \pm}(\alpha, z)$ makes much more difficult to obtain the new critical points $\alpha_{0}^{( \pm)}, z_{0}^{( \pm)}$ minimizing the corresponding energy surface. Instead of carrying out a numerical computation of $\alpha_{0}^{( \pm)}, z_{0}^{( \pm)}$for different values of $j$ and $\lambda$, we shall use the approximation $\alpha_{0}^{( \pm)} \approx \alpha_{0}, z_{0}^{( \pm)} \approx z_{0}$, which turns out to be quite good except in a close neighborhood around $\lambda_{c}$ which diminishes as the number of particles $N=2 j$ increases (see Figure 4 for the symmetric case and Ref. [48]). With this approximation, we expect a rather good agreement between our numerical and variational results except perhaps in a close vicinity of $\lambda_{c}$.

In order to compute uncertainty relations for information entropies in position and momentum representations, we shall make use of the Holstein-Primakoff representation (9). Redefining $\beta \equiv \sqrt{2 j} z$, it can be seen (see e.g. $[49,50]$ ) that spin- $j$ coherent states $|z\rangle$ go over to ordinary coherent states $|\beta\rangle \equiv e^{|\beta|^{2} / 2} e^{\beta b^{\dagger}}|0\rangle$ for $j \gg 1$ (when identifying $|j,-j\rangle \equiv|0\rangle$ ). Thus, we shall assume the approximation:

$$
|z\rangle \simeq|\beta\rangle,
$$

which turns out to be a quite good estimate even for relatively small values of $j$. Introducing position and momentum operators for the two bosonic modes as in (10), and taking into account the position and momentum representation of an ordinary (canonical) $\operatorname{CS}\left(\alpha=\alpha_{1}+i \alpha_{2}\right)$ [50]:

$$
\begin{aligned}
& \langle x \mid \alpha\rangle=\left(\frac{\omega}{\pi}\right)^{1 / 4} e^{i \sqrt{2 \omega} \alpha_{2} x} e^{-\left(\sqrt{\omega} x-\sqrt{2} \alpha_{1}\right)^{2} / 2}, \\
& \langle p \mid \alpha\rangle=\left(\frac{\omega}{\pi}\right)^{1 / 4} e^{i \sqrt{\frac{2}{\omega}} \alpha_{1} p} e^{-\left(\frac{p}{\sqrt{\omega}}-\sqrt{2} \alpha_{2}\right)^{2} / 2},
\end{aligned}
$$

the explicit expression of the ground state wave function $\left|\alpha_{0}, \beta_{0}\right\rangle_{+}$in position $\left(\psi(x, y)=\left\langle x, y \mid \alpha_{0}, \beta_{0},+\right\rangle\right)$ and momentum $\left(\phi\left(p_{x}, p_{y}\right)=\left\langle p_{x}, p_{y} \mid \alpha_{0}, \beta_{0},+\right\rangle\right)$ representations 
$\frac{1}{N} \partial_{q} H_{+}\left(\alpha_{0}, z_{0}\right)$

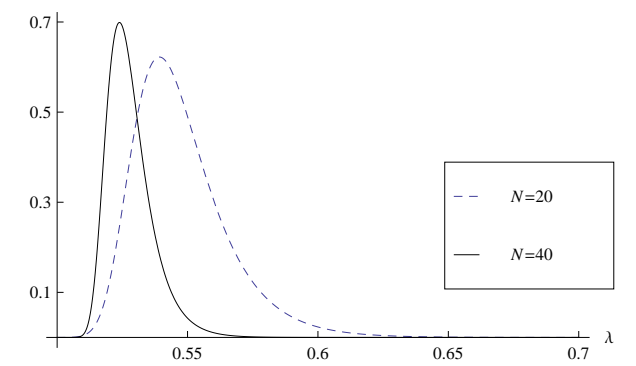

$\frac{1}{N} \partial_{\theta} H_{+}\left(\alpha_{0}, z_{0}\right)$

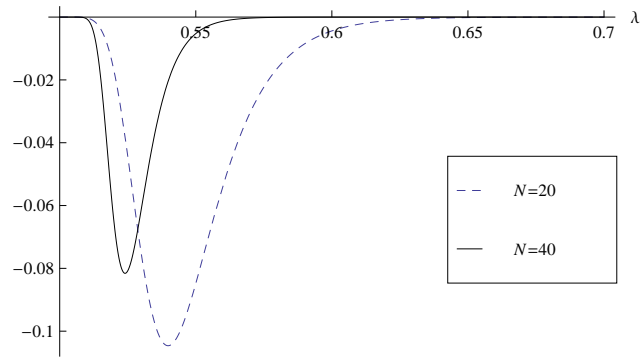

FIG. 4. Derivative of the mean energy $\mathcal{H}_{+}(\alpha, z)$ per particle with respect to $q=\sqrt{2} \operatorname{Re}(\alpha)$ and $\theta=2 \arctan (|z|)$, evaluated at the critical points (16), as a function of $\lambda$ for $N=20$ (dashed line) and $N=40$ (solid line) particles and $\omega_{0}=\omega=$ 1 .

can be easily obtained as:

$$
\begin{aligned}
& \psi(x, y)=\mathcal{N}_{+}\left(e^{-\frac{1}{2}\left(\sqrt{\omega} x-\sqrt{2} \alpha_{0}\right)^{2}-\frac{1}{2}\left(\sqrt{\omega_{0}} y-\sqrt{2} \beta_{0}\right)^{2}}\right. \\
& \left.+e^{-\frac{1}{2}\left(\sqrt{\omega} x+\sqrt{2} \alpha_{0}\right)^{2}-\frac{1}{2}\left(\sqrt{\omega_{0}} y+\sqrt{2} \beta_{0}\right)^{2}}\right), \\
& \phi\left(p_{x}, p_{y}\right)=2 \mathcal{N}_{+} e^{-\frac{p_{x}^{2}}{2 \omega}-\frac{p_{y}^{2}}{2 \omega_{0}}} \cos \left(\sqrt{2}\left(\frac{p_{x}}{\sqrt{\omega}} \alpha_{0}+\frac{p_{y}}{\sqrt{\omega_{0}}} \beta_{0}\right)\right),
\end{aligned}
$$

where now $\mathcal{N}_{+}\left(\alpha_{0}, \beta_{0}\right)=\left(\frac{2 \pi}{\omega_{0} \omega}\left(1+e^{-2 \alpha_{0}^{2}-2 \beta_{0}^{2}}\right)\right)^{-1 / 2}$ is a new normalization factor. Note that $\psi$ and $\phi$ depend on $j$ and $\lambda$ through $\alpha_{0}$ and $\beta_{0}$. Moreover, note also that for $\lambda>\lambda_{c}$ the ground-state density function $\rho(x, y)=|\psi(x, y)|^{2}$ splits up into two Gaussian packets centered at antipodal points $\sqrt{2}\left(\alpha_{0}, \beta_{0}\right)$ and $-\sqrt{2}\left(\alpha_{0}, \beta_{0}\right)$ in the $x-y$ plane. The packets move away from each other for increasing $j$ above the critical point $\lambda>\lambda_{c}$. In momentum space, $\gamma\left(p_{x}, p_{y}\right)=\left|\phi\left(p_{x}, p_{y}\right)\right|^{2}$ is a Gaussian modulated by a cosine function which oscillates rapidly for high $j$ for $\lambda>\lambda_{c}$. This behavior is also captured by the numerical solution as depicted in Figure 2.

This particular ground state wave function structure leads to a Heaviside (step) function behavior of the Renyi entropy in position

$$
R_{\rho}^{\mu}= \begin{cases}\ln \left(\mu^{\frac{1}{\mu-1}} \pi\right), & \text { if } \lambda<\lambda_{c} \\ \ln \left(2 \mu^{\frac{1}{\mu-1}} \pi\right), & \text { if } \lambda \geq \lambda_{c}\end{cases}
$$
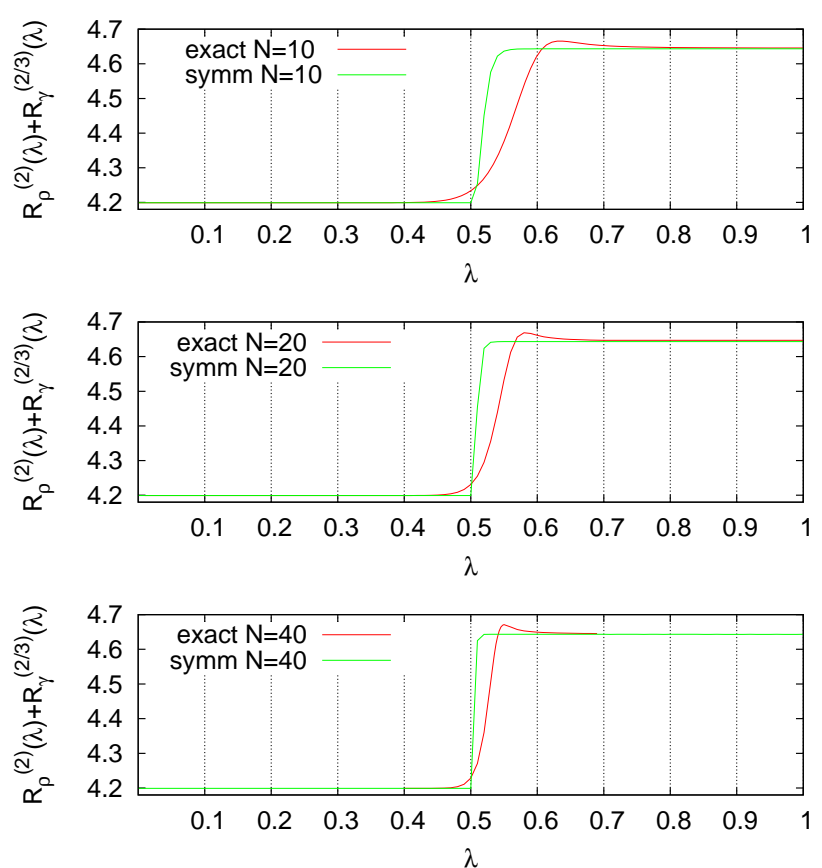

FIG. 5. Analytical approximation (green line) and numerical (exact) results (red line) for the ground state Rényi uncertainty entropy sum $R_{\rho}^{\mu}+R_{\gamma}^{\nu}$ for for $N=10$ (top panel), 20 (middle panel) and 40 (bottom panel) and $\omega_{0}=\omega=1$.

and momentum

$$
R_{\gamma}^{\nu}= \begin{cases}\ln \left(\nu^{\frac{1}{\nu-1}} \pi\right), & \text { if } \lambda<\lambda_{c} \\ \ln \left(\left(\frac{\Gamma(2 \nu+1)}{\Gamma^{2}(\nu+1) 2^{\nu} \nu}\right)^{\frac{1}{1-\nu}} \pi\right), & \text { if } \lambda \geq \lambda_{c}\end{cases}
$$

representations in the thermodynamic limit $(j \rightarrow \infty)$. This behavior can be inferred from Figure 5 . In the normal phase, the inequality (3) saturates (that is, the total entropy is exactly $g(\mu, \nu))$ because the ground state wave function (23) is a Gaussian centered at the origin in position and momentum representation. Above the critical point $\lambda_{c}$, the original Gaussian wave packet splits up into two subpackets with negligible overlap, which results in a sudden rise of the total Renyi's entropy. In the limit $\mu, \nu \rightarrow 1$, we recover the expression for the Shannon entropy given in [32]

$$
S_{\rho}+S_{\gamma}= \begin{cases}S^{\text {normal }}=\ln (e \pi)^{2} \simeq 4.29, & \text { if } \lambda<\lambda_{c} \\ S^{\text {super }}=\ln \left((2 \pi)^{2} e\right) \simeq 4.68, & \text { if } \lambda \geq \lambda_{c}\end{cases}
$$

We would also like to point out that the Heaviside (step) function behavior of $R_{\rho}^{\mu}+R_{\gamma}^{\nu}$ should also appear in other quantum systems where a single wavepacket splits up into several subpackets above a critical value $\lambda_{c}$ of some parameter $\lambda$ of the theory. In particular, for $M$ identical subpackets with negligible overlap, one can see that the Rényi entropy in position representation $R_{\rho}^{\mu}$ increases by an amount of $\ln (M)$. 
(a)

These analytical expressions are in agreement with our

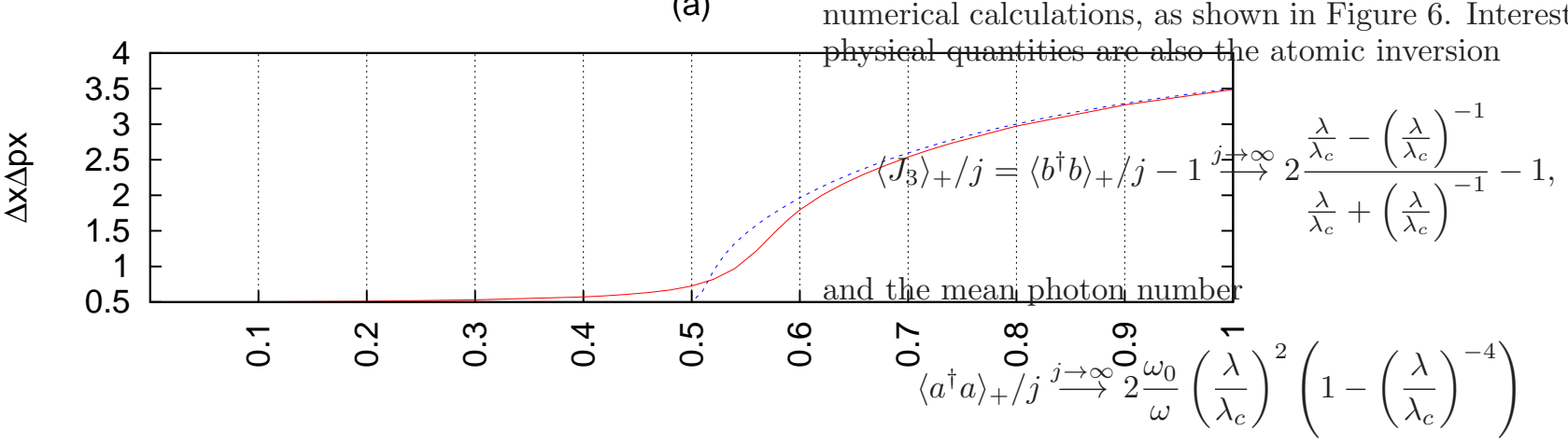

(b)

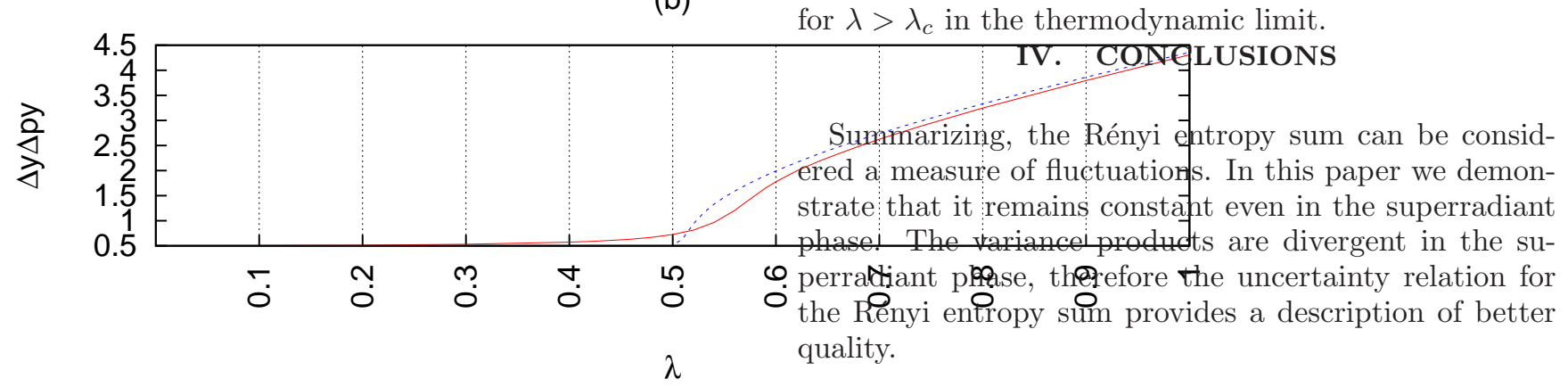

FIG. 6. Analytical approximation (dotted-blue line) and numerical results (solid-red line) for (a) variance products $\Delta x \Delta p_{x}$, and (b) $\Delta y \Delta p_{y}$ for the ground-state for $j=10$ and $\omega_{0}=\omega=1\left(\lambda_{c}=0.5\right)$.

For completeness, we also give the explicit expressions for expectation values

$$
\begin{aligned}
& \langle a\rangle_{+}=\langle b\rangle_{+}=\left\langle a^{\dagger}\right\rangle_{+}=\left\langle b^{\dagger}\right\rangle_{+}=0, \\
& \left\langle a^{\dagger} a\right\rangle_{+}=\alpha_{0}^{2}\left(4 \pi \mathcal{N}_{+}^{2}-1\right), \\
& \left\langle b^{\dagger} b\right\rangle_{+}=\beta_{0}^{2}\left(4 \pi \mathcal{N}_{+}^{2}-1\right),
\end{aligned}
$$

and fluctuations

$$
\begin{aligned}
& \Delta x=\sqrt{\frac{4 \pi \mathcal{N}_{+}^{2} \alpha_{0}^{2}+\frac{1}{2}}{\omega}}, \quad \frac{\Delta p_{x}}{\sqrt{\omega}}=\sqrt{2 \alpha_{0}^{2}\left(2 \pi \mathcal{N}_{+}^{2}-1\right)+\frac{1}{2}}, \\
& \Delta y=\sqrt{\frac{4 \pi \mathcal{N}_{+}^{2} \beta_{0}^{2}+\frac{1}{2}}{\omega_{0}}}, \quad \frac{\Delta p_{y}}{\sqrt{\omega_{0}}}=\sqrt{2 \beta_{0}^{2}\left(2 \pi \mathcal{N}_{+}^{2}-1\right)+\frac{1}{2}} .
\end{aligned}
$$

\section{ACKNOWLEDGMENTS}

This work was supported by the Projects: PYR2010-24 (Proyecto GENIL ref. CEB09-0010), FIS200801143 and FIS2008-06078-C03-01 (Spanish MICINN), 08814/PI/08 (Fundación Séneca), FQM-165/0207 and FQM219 (Junta de Andalucía). The work is also supported by the TAMOP 4.2.1/B-09/1/KONV-2010-0007 and the TAMOP $4.2 .2 / \mathrm{B}-10 / 1-2010-0024$ projects. The project is co-financed by the European Union and the European Social Fund. Grant OTKA No. K 100590 is also gratefully acknowledged.
[1] I. I. Hirschman, Am. J. Math. 79, 152 (1957)

[2] Bialynicki-Birula, I., Mycielski, J.: Commun. Math. Phys. 44, 129 (1975)

[3] W. Beckner, Ann. Math. 102, 159 (1975)

[4] I. Bialynicki-Birula, Phys. Lett. A 103, 253 (1984)

[5] H. Maassen, J. B. M. Uffink, Phys. Rev. Lett. 60, 1103 (1988)

[6] J. Sánchez-Ruiz, Phys. Lett. A 244, 189 (1998)

[7] I. Bialynicki-Birula, Phys. Rev. A 74, 052101 (2006)

[8] E. Romera, F. de los Santos Phys. Rev. Lett.99, 263601 (2007); Phys Rev. A 78, 013837 (2008).
[9] T. Schürmann and I. Hoffmann Found Phys 39, 958 (2009).

[10] S. R. Gadre, Phys. Rev. A 30, 620 (1984).

[11] S. R. Gadre, S. B. Sears, S. J. Chakrovarty and R. D. Bendale Phys. Rev. A 32, 2602 (1985).

[12] S. Sachdev, Quantum Phase Transitions, Cambridge University Press (2000).

[13] A. Rényi, Proceedings of the 4th Berkeley Symposium on Mathematical Statistics and Probability, Volume 1: Contributions to the Theory of Statistics (1961) 547. 
[14] C.E. Shannon, A mathematical theory of communication, Bell. Sys. Tech. J. 27 (1948) 379; ibid. (1948) 623.

[15] O. Gühne and M. Lewenstein, Phys. Rev. A 70, 022316 (2004).

[16] R. Renner, N. Gisin and B. Kraus, Phys. Rev. A 72 $012332(2005)$.

[17] P. Lévay, S. Nagy and J. Pipek, Phys. Rev. A 72, 022302 (2005).

[18] D. G. Arbo, C. O. Reinhold, J. Burgdörfer, A. K. Pattanayak, C. L. Stokely, W. Zhao, J. C. Lancaster and F. B. Dunning, Phys. Rev. A 67, 063401 (2003).

[19] E. Romera and F. de los Santos, Phys. Rev. A, 78, 013837- (2008).

[20] E. Romera and Á. Nagy, Phys. Lett. A 3724918 (2008).

[21] E.. Romera and Á. Nagy, Phys. Lett. A 372, 6823 (2008).

[22] Á. Nagy and E. Romera, Phys. Lett. A 373844 (2009).

[23] Á. Nagy and E. Romera, Int. J. Quantum Chem. 109 2490 (2009).

[24] H. Maassen and J. B. M. Uffink, Phys. Rev. Lett. 60, 1103 (1988).

[25] A. Dembo, T. M. Cover and J. A. Thomas, IEEE Trans. Inf. Theory 37, 1501 (1991).

[26] I. Bialynicki-Birula, Phys. Rev. A 74, 052101 (2006).

[27] W. Beckner, Ann. Math. 102, 159, (1975).

[28] I. I. Hirschmann, Am. J. Math. 79, 152 (1957).

[29] I. Bialynicki-Birula and J. Mycielski, Commun. Math. Phys. 44, 129 (1975).

[30] I. Hornyák and Á. Nagy, Int. J. Quantum Chem. 112, 1285 (2012).

[31] J. J. Halliwell, Phys. Rev. D 48, 2739 (1993).

[32] E. Romera, M. Calixto and Á. Nagy, Europhys. Lett. 97, 20011 (2012).
[33] E. Majerníková, V. Majerník and S. Shpyrko, Eur. Phys. J. B. 38, 25 (2004).

[34] R. H. Dicke, Phys. Rev. 93, 99 (1954).

[35] K. Hepp and E. H. Lieb, Phys. Rev. A 8, 2517 (1973).

[36] Y. K. Wang and F. T. Hioe, Phys. Rev. A 7, 831 (1973)

[37] H. J. Carmichael, C. W. Gardiner, and D. F. Walls, Phys. Lett. A 46, 47 (1973).

[38] C. Emary and T. Brandes, Phys. Rev. E 67, 066203 (2003).

[39] C. Emary and T. Brandes, Phys. Rev. Lett. 90, 044101 (2003).

[40] N. Lambert, C. Emary, and T. Brandes, Phys. Rev. Lett. 92, 073602 (2004).

[41] K. Baumann, C. Guerlin, F. Brennecke and T. Esslinger, Nature 464, 1301 (2010).

[42] K. Baumann, R. Mottl, F. Brennecke, T. Esslinger, arXiv:1105.0426v1 (2011).

[43] E. Romera and Á. Nagy, Phys. Lett. A 3753066 (2011).

[44] E. Romera, K. Sen and Á. Nagy, J.Stat. Mech. doi:10.1088/1742-5468/2011/09/P09016

[45] Miguel A. Bastarrachea-Magnani, Jorge G. Hirsch Numerical solutions of the Dicke Hamiltonian arxiv:1108.0703 (2011)

[46] T. Holstein and H. Primakoff, Phys. Rev. 58, 1098 (1940).

[47] O. Castaños, E. Nahmad-Achar, R. López-Peña and J. G. Hirsch, Phys. Rev. A 83, 051601 (2011).

[48] O. Castaños, E. Nahmad-Achar, R. López-Peña, and J. G. Hirsch, Phys. Rev. A, 84013819 (2011).

[49] J.M. Radcliffe, J. Phys. A 4, 313 (1971).

[50] A. Perelomov, Generalized Coherent States and Their Applications, Springer-Verlag (1986). 